José Carlos Bermejo Higuera ${ }^{1}$

Marta Villacieros Durbán²

Rosa Carabias Maza²

Bernardino Lozano González ${ }^{3}$

1. Dirección. Centro Humanización de la Salud. Tres Cantos. Madrid.

2. Investigación. Centro Humanización de la Salud. Tres Cantos. Madrid.

3. EAPS. Centro Humanización de la Salud. Tres Cantos. Madrid.

Correspondencia:

Marta Villacieros Durbán

Centro Humanización de la Salud. Tres Cantos

Sector Escultores 39

28760 Tres Cantos (Madrid)

Tel.: 918060696

E-mail: investigacion@humanizar.es

\section{Niveles y motivos de satisfacción o insatisfacción en los familiares de los fallecidos en una unidad de cuidados paliativos}

\author{
Levels and reasons for satisfaction or \\ dissatisfaction in the relatives of the \\ deceased in a palliative care unit
}

\section{RESUMEN}

Objetivo: conocer el nivel y motivos de satisfacción o insatisfacción de los familiares de fallecidos a lo largo del 2011 en una unidad de cuidados paliativos (UCP) de la Comunidad de Madrid.

Método: estudio descriptivo, por autoinforme anónimo y voluntario enviado mediante correo postal, que incluía variables demográficas del encuestado (familiar), su relación con el fallecido, 9 preguntas cerradas relativas a la calidad de la atención recibida, valoración global de la atención recibida, ambiente percibido en el equipo de UCP y tres preguntas abiertas sobre motivos de satisfacción o insatisfacción. Resultados: respondieron 66 personas al cuestionario, con una media de edad de 59,02 años. La tasa de respuesta fue del 27,5\%. Un 28,8\% (19) hombres y un $71,2 \%$ (47) mujeres, con estudios. El fallecido era progenitor en un 56,1\% y cónyuge en un 40,9\%. La valoración global media fue de 9,11.

Entre el $90 \%$ y el $98,5 \%$ contestaron que el dolor de su familiar se controló bien, su familiar tuvo buena calidad de vida antes de morir, su muerte fue tranquila y en paz, tuvo la suficiente ayuda del equipo para enfrentarse con la enfermedad y la muerte, tuvo facilidades para hablar con cualquier miembro del equipo, y se sintió satisfecho con la información recibida sobre la enfermedad y su evolución.

El 50,8\% creía que no tenía temas pendientes, el 29,2\% que recibió ayuda en este sentido, y el 15,4\% no la recibió. El 74,6\% está elaborando

el duelo adecuadamente; el 88,9\% contestó que la coordinación con este otro equipo (de atención al duelo) se realizó de forma adecuada. El ambiente del equipo se valoró principalmente como acogedor y amable. Los motivos de satisfacción hacen referencia al trato recibido "exquisito, aseo, comida, amabilidad y empatía, delicadeza, calor humano y voluntarios" y, en general, "las instalaciones". Los de insatisfacción, a la "falta de información del médico y la atención de distintos profesionales (psicólogo, fisioterapeuta, trabajador social y sacerdote)". Conclusiones: destacamos que la asistencia integral incluye los aspectos más humanos y propios de la esfera íntima; las personas en situación de vulnerabilidad los agradecen o echan de menos cuando no los reciben.

PALABRAS CLAVE: cuidados paliativos, cuidador principal, encuesta de satisfacción, control de calidad asistencial.

\section{ABSTRACT}

Objective: to know level and reasons for satisfaction or dissatisfaction in the relatives of deceased throughout 2011 in a CPU from the community of Madrid.

Method: descriptive study, by anonymous and voluntary selfreport sent via postal mail, including demographic variables of the respondent (relatives), their relationship with the deceased, 9 closed questions relating to the quality of care received, overall assessment of care received, environment perceived in the CPU and 3 open questions on reasons for satisfaction or dissatisfaction.

Results: 66 people answered to the questionnaire with mean age 59,02 . The response rate was $27.5 \%$. 28.8\% (19) men and $71.2 \%$ (47) women, with studies. The deceased was parent $56.1 \%$ and spouse by $40.9 \%$. The average overall score was 9.11 . Between $90 \%$ and $98.5 \%$ answered that his relative pain was well controlled, had a good quality of life before dying, his death was peaceful and in peace, had sufficient support team to deal with the illness and death, had facilities to speak to any member of the team and felt satisfied with the information received about the disease and its evolution. $50.8 \%$ believed that he had no outstanding issues, 29.2\% who received assistance in this regard, $15.4 \%$ not received. $74.6 \%$ is preparing the grief properly, $88.9 \%$ replied that coordination with the other (grief support) team was proper. The atmosphere of the team was mainly assessed how welcoming and friendly.

The reasons for satisfaction make reference to the treat "exquisite, toilet, food, friendliness and empathy, delicacy, voluntary and human heat" and general "facilities". Dissatisfaction to the "lack of information from the physician and the attention of various professionals (psychologist, physiotherapist, social worker and priest).

Conclusions: we highlight the overall assistance includes aspects of the human and intimate sphere, vulnerable people appreciate them or miss when they do not receive them.

KEYWORDS: Palliative care, main carer, satisfaction survey, health care quality control. 


\section{- INTRODUCCIÓN}

Uno de los signos de humanización de la medicina es el avance de la reflexión y la práctica de los cuidados paliativos. Estos constituyen una señal relevante de que los hombres nos preocupamos no solo de curar a quien está enfermo, sino también de cuidar a quien no puede ser curado. Los que trabajamos en ello observamos que dentro de los cuidados paliativos son muchas las necesidades que hay que atender. Consideramos, pues, que es el usuario, debido al momento de fragilidad en el que se encuentra, el que ha de valorar si dichos cuidados y atención están siendo de calidad o cubriendo sus expectativas.

Distintas organizaciones, como la Sociedad Española de Cuidados Paliativos (SECPAL) $)^{1}$ y el Centro de Humanización de la salud (CEHS) ${ }^{2}$, trabajan de manera continuada para contribuir a la sensibilización y formación de los profesionales del ámbito de los cuidados paliativos, de modo que los propios equipos se hagan con las riendas de la mejora continua de la calidad. Se promueven estudios que analizan la calidad del cuidado y el grado de satisfacción con la atención recibida en las UCP espańolas ${ }^{3-5}$ que, como muchos autores expresan, están sometidos a un influjo multifactorial, por parte tanto de personas como de situaciones o instalaciones, como son la situación emocional del doliente, la naturaleza y calidad de la relación familiar-enfermo, la complejidad clínica-emocional-social-espiritual del paciente, el nivel previo de información, las expectativas, el trato recibido con anterioridad y la duración de la intervención ${ }^{6-8}$.

Sobre las causas de sufrimiento del paciente, se ha visto su relación con la percepción subjetiva del paso del tiempo" con el conocimiento de la enfermedad ${ }^{10}$, con el aburrimiento, con el control sintomático, con la capacidad para realizar actividades distractoras y con el estado de ánimo. Pero para muchos pacientes la muerte próxima no es la principal amenaza, sino los factores cambiantes ${ }^{11,12}$.

Anualmente se recogen encuestas de satisfacción en nuestras unidades $^{3-5} \mathrm{o}$ en domicilios ${ }^{13}$, para contrastar o mejorar la atención ofertada. Todas ellas se centran en preguntas sociales, cuestiones de relación y situación familiar, control de síntomas, soporte emocional, información, comunicación, acceso al equipo asistencial, resolución de asuntos pendientes o valoración global de la atención recibida. Cuando pasa el tiempo desde el fallecimiento, también indaga acerca de la elaboración del duelo ${ }^{3,4}$, y cuando el cuidado es a domicilio, también son importantes las preguntas de carácter económico ${ }^{13}$.

Considerando esta información en su globalidad fuente de calidad en la atención, el objeto de este estudio fue conocer el nivel y motivos de satisfacción o insatisfacción de los usuarios de una UCP de la Comunidad de Madrid. Para ello enviamos un cuestionario ad hoc a los familiares que fueron usuarios de la unidad durante un ańo completo, y para poder recoger medidas cuantitativas donde la mayoría se agrupen sin sacrificar la idiosincrasia de cada uno (sentimientos, pensamientos, percepciones y conductas o actitudes de otros que influyen) nos basamos en encuestas ya utilizadas ${ }^{3-5}$ a las que añadimos preguntas abiertas.

\section{- METODOLOGÍA}

Diseño: estudio descriptivo, por autoinforme anónimo y voluntario enviado mediante correo postal.

Participantes: de los 240 familiares de fallecidos durante el ańo previo al estudio (2011) en una UCP de la Comunidad de Madrid, respondieron al cuestionario 66 personas. La tasa de respuesta fue del 27,5\%.

Instrumento: se adaptó el cuestionario de satisfacción de Palomar y cols. ${ }^{3}$ para el caso de una UCP. Se recogieron variables demográficas del encuestado, nivel de estudios, relación con el fallecido, y tiempo desde el fallecimiento, 9 preguntas cerradas, relativas a la calidad de la atención recibida (control de síntomas, soporte emocional recibido, ayuda recibida, información y comunicación, disponibilidad de acceso al equipo asistencial, resolución de asuntos pendientes y necesidad y coordinación con otros recursos) y se pidió valoración global de la atención recibida (mediante escala visual del 1 al 10). También se solicitó valoración del ambiente percibido en el equipo de UCP (con 8 expresiones positivas y negativas a escoger una o más respuestas). Finalmente se sustituyó el apartado de quejas y sugerencias por tres preguntas abiertas que, a nuestro juicio, centraban mejor las respuestas sobre motivos de satisfacción o insatisfacción durante la estancia en UCP: lo que más le ayudó, lo que echó de menos y lo que le gustaría que encontraran otros al ingresar.

Procedimiento: previo a las Jornadas de Cuidados Paliativos de 2012, junto con la invitación a las mismas, se envió el cuestionario de satisfacción a todos los familiares, a responder en sobre prefranqueado y de manera anónima. Se enviaron cartas a los 240 familiares de fallecidos durante el año previo, 2011, y se les pidió su colaboración contestando y enviando la encuesta por correo postal.

Análisis estadístico: descriptivo y previa categorización de respuestas en las preguntas abiertas.

La investigación fue revisada por el Comité de Ética Asistencial de la institución y con los principios de la declaración de Helsinki.

\section{- RESULTADOS}

La media de edad de los 66 familiares que respondieron fue de 59,02 años (desviación típica: 12,6; mediana: 61 años). El familiar había fallecido de media hacía 6,88 meses (desviación típica: 3; mediana: 6,50).

Los respondedores en un 28,8\% (19) eran hombres y en un 71,2\% (47), mujeres; a partes iguales, $32,3 \%$ con estudios primarios (21), secundarios (21) y superiores (21). Solo un 3,1\% (2 personas) no tenía estudios.

El fallecido era progenitor en un $56,1 \%$ (37) y el cónyuge en un $40,9 \%$ (27) de los casos. Un 3\% (2) marcaron otro grado.

El 93,8\% contestó que el dolor de su familiar se controló bien. El 92,3\%, que su familiar tuvo buena calidad de vida antes de morir. El $90,8 \%$, que su muerte fue tranquila y en paz. El $93,8 \%$, que tuvo la suficiente ayuda del equipo para enfrentarse con la enfermedad y la muerte. El 98,5\%, que tuvo facilidades para hablar con cualquier miembro del equipo (tabla 1).

Respecto a la ayuda para resolver temas pendientes (testamentos, tutorías legales, tramitación de pensiones o pagas, celebraciones familiares, ilusión para hacer cosas, etc.), el 50,8\% creía que no tenía temas pendientes; el 29,2\%, que recibió ayuda en este sentido; el 15,4\%, no la recibió, y casi un $5 \%$ no sabe o no contesta (v. tabla 1).

El 92,3\% se sintió satisfecho con la información recibida sobre la enfermedad y su evolución. El 74,6\% está elaborándolo el duelo adecuadamente, el 9,5\% no, y casi el $16 \%$ no sabe o no contesta. El $88,9 \%$ contestó que la coordinación con este otro equipo (de atención al duelo) se realizó de forma adecuada, y el $11,1 \%$ no sabe no contesta (v. tabla 1 ).

La media de la valoración de la atención global recibida fue de 9,11, aunque el $61,5 \%$ de los respondedores valoraron la atención recibida como 10.

El ambiente de nuestro equipo fue valorado por el $80,3 \%$ como acogedor, por el $77,3 \%$ como amable, por el $68,2 \%$ como tranquilizador y por el 33,3\% como simpático. También hubo personas que lo percibieron triste (un 4,5\%), un 1,5\% que lo sintieron áspero y frío, y un $3 \%$ sintió que era un ambiente angustiante (v. tabla 1).

Las respuestas a las preguntas abiertas se detallan en la figura $1 \mathrm{y}$ se resumen a continuación. 
Tabla 1. Porcentajes de respuesta a las preguntas cerradas sobre atención percibida y valoración del ambiente del equipo

\begin{tabular}{|c|c|c|c|c|c|c|c|c|}
\hline \multirow{2}{*}{ Preguntas del cuestionario } & \multicolumn{8}{|c|}{ Porcentaje (recuento) } \\
\hline & \multicolumn{2}{|c|}{ Sí } & \multicolumn{2}{|c|}{ No } & \multicolumn{2}{|c|}{ No sabe/no contesta } & \multicolumn{2}{|c|}{ Otras opciones } \\
\hline ¿Cree que el dolor de su familiar se controló bien? & \multicolumn{2}{|c|}{$93,8(61)$} & \multicolumn{2}{|c|}{$3,1(2)$} & \multicolumn{2}{|c|}{$1,5(1)$} & \multicolumn{2}{|c|}{ 1,5 (1) no tenía dolor } \\
\hline $\begin{array}{l}\text { ¿Cree que su familiar tuvo buena calidad de vida } \\
\text { antes de morir? }\end{array}$ & \multicolumn{2}{|c|}{$92,3(60)$} & \multicolumn{2}{|c|}{$4,6(3)$} & \multicolumn{2}{|c|}{$3,1(2)$} & & \\
\hline ¿Cree que su muerte fue tranquila y en paz? & \multicolumn{2}{|c|}{$90,8(59)$} & \multicolumn{2}{|c|}{$6,2(4)$} & \multicolumn{2}{|c|}{$3,1(2)$} & & \\
\hline $\begin{array}{l}\text { Usted, como cuidador del paciente, ¿tuvo la } \\
\text { suficiente ayuda del equipo para enfrentarse con la } \\
\text { enfermedad y la muerte? }\end{array}$ & \multicolumn{2}{|c|}{$93,8(60)$} & \multicolumn{2}{|c|}{$6,2(4)$} & \multicolumn{2}{|c|}{$1,6(1)$} & \multicolumn{2}{|c|}{ 1,6 (1) no la necesitaba } \\
\hline $\begin{array}{l}\text { ¿Tenían ustedes facilidades para hablar con } \\
\text { cualquier miembro del equipo? }\end{array}$ & \multicolumn{2}{|c|}{$98,5(64)$} & \multicolumn{2}{|c|}{$1,5(1)$} & \multicolumn{2}{|c|}{0} & & \\
\hline $\begin{array}{l}\text { ¿Se les facilitó ayuda parar resolver temas } \\
\text { pendientes que ustedes pudieran tener } \\
\text { (testamentos, tutorías legales, tramitación de } \\
\text { pensiones o pagas, celebraciones familiares, ilusión } \\
\text { para hacer cosas, etc.) }\end{array}$ & \multicolumn{2}{|c|}{$29,2(19)$} & \multicolumn{2}{|c|}{$15,4(10)$} & \multicolumn{2}{|c|}{$4,6(3)$} & \multicolumn{2}{|c|}{$\begin{array}{l}50,8 \text { (33) no tenían } \\
\text { temas pendientes }\end{array}$} \\
\hline $\begin{array}{l}\text { Tanto el paciente como usted, ¿estaban satisfechos } \\
\text { con la información recibida sobre la enfermedad y } \\
\text { su evolución? }\end{array}$ & \multicolumn{2}{|c|}{$92,3(60)$} & \multicolumn{2}{|c|}{$3,1(2)$} & \multicolumn{2}{|c|}{$4,6(3)$} & & \\
\hline $\begin{array}{l}\text { ¿Cree usted que está elaborando adecuadamente } \\
\text { el duelo? }\end{array}$ & \multicolumn{2}{|c|}{$74,6(47)$} & \multicolumn{2}{|c|}{$9,5(6)$} & \multicolumn{2}{|c|}{$15,9(10)$} & & \\
\hline $\begin{array}{l}\text { ¿Cree usted que la coordinación con este otro } \\
\text { equipo se realizó de forma adecuada? }\end{array}$ & \multicolumn{2}{|c|}{$88,9(56)$} & \multicolumn{2}{|c|}{$11,1(7)$} & \multicolumn{2}{|c|}{0} & & \\
\hline \multirow{2}{*}{$\begin{array}{l}\text { Marque con una cruz todas las características que } \\
\text { usted crea que puedan definir el ambiente de } \\
\text { nuestro equipo: }\end{array}$} & Acogedor & Triste & Áspero & Tranquilizador & Amable & Angustiante & Frío & Simpático \\
\hline & $80,3(53)$ & $4,5(3)$ & $1,5(1)$ & $68,2(45)$ & $77,3(51)$ & $3,0(2)$ & $1,5(1)$ & $33,3(22)$ \\
\hline
\end{tabular}

- ¿Qué o quién fue lo que más le ayudó durante el ingreso de su ser querido?

La respuesta más común (un 35\%) se pudo agrupar en atención recibida por el personal en general y las instalaciones, a continuación casi el $18 \%$ hicieron referencia al personal de enfermería y los auxiliares, después "el médico" (14,5\%), "el sacerdote, la atención espiritual, la fe y la presencia" (14\%), "otro personal como recepción, gerente, limpieza, trabajador social” (10\%), "el psicólogo" (9\%), y por último familiares y amigos" (1\%).

- ¿Qué o a quién echó de menos por parte de nuestro equipo?

El $75 \%$ de las personas se agruparían en "nada ni a nadie, todo fue correcto, tuvimos cariño cuidados y libertad". El 21\% echó de menos "la información del médico, la atención psicológica, un fisioterapeuta, trabajador social y sacerdote". El 3\% "otros servicios como el funerario" o no le gustó el ambiente de tristeza.

- ¿Qué querría que encontraran otros al ingresar en esta unidad de cuidados paliativos?

El 67\% aludieron de distintas maneras a "lo mismo que yo encontré, la buena atención en todos los sectores, el trato recibido fue exquisito, aseo, comida, amabilidad y empatía”. El 23\% querrían que otros encontraran "mejor atención, información, delicadeza, calor humano y voluntarios" y el 10\% echó de menos "la intimidad en las habitaciones y más luz".

\section{- DISCUSIÓN}

Nuestro cuestionario, al igual que en otros estudios ${ }^{3,4}$, recoge una valoración global muy satisfactoria del familiar sobre la estancia pasada en UCP (media de 9,11).

El perfil del familiar que responde, también en la línea de lo recogido en otros estudios ${ }^{3}$, es el de una mujer con estudios, de unos 59 ańos, cuyo padre/madre (o también podría ser esposo) falleció hace ya 7 meses. Cree que el dolor de su familiar se controló bien, que tuvo buena calidad de vida antes de morir, que su muerte fue tranquila y en paz, que tuvo la suficiente ayuda del equipo de la unidad para enfrentarse con la enfermedad y la muerte, que tuvo facilidades para hablar con cualquier miembro del equipo y se sintió satisfecha con la información recibida sobre la enfermedad y su evolución. Además siente que está elaborando bien el duelo y que la coordinación con ese otro equipo se ha realizado adecuadamente.

Todas estas puntuaciones, si bien son un poco más elevadas que los datos recogidos en el estudio de referencia (94\% frente a 94\%; $92 \%$ frente $82 \%$; $91 \%$ frente a $82 \%$; $94 \%$ frente a $79 \%$; $98 \%$ frente a $81 \%$; $92 \%$ frente a $84 \% ; 75 \%$ frente a $66 \%$ ), también es cierto que podrían estar sesgados por una tasa de respuesta inferior (casi 30\% frente a 40\%).

Aun así, parece que los datos configuran patrones paralelos. No solo las respuestas descritas, sino también las distribuciones de respuesta para la pregunta sobre temas pendientes, sujeta a mayor dispersión, siguen 


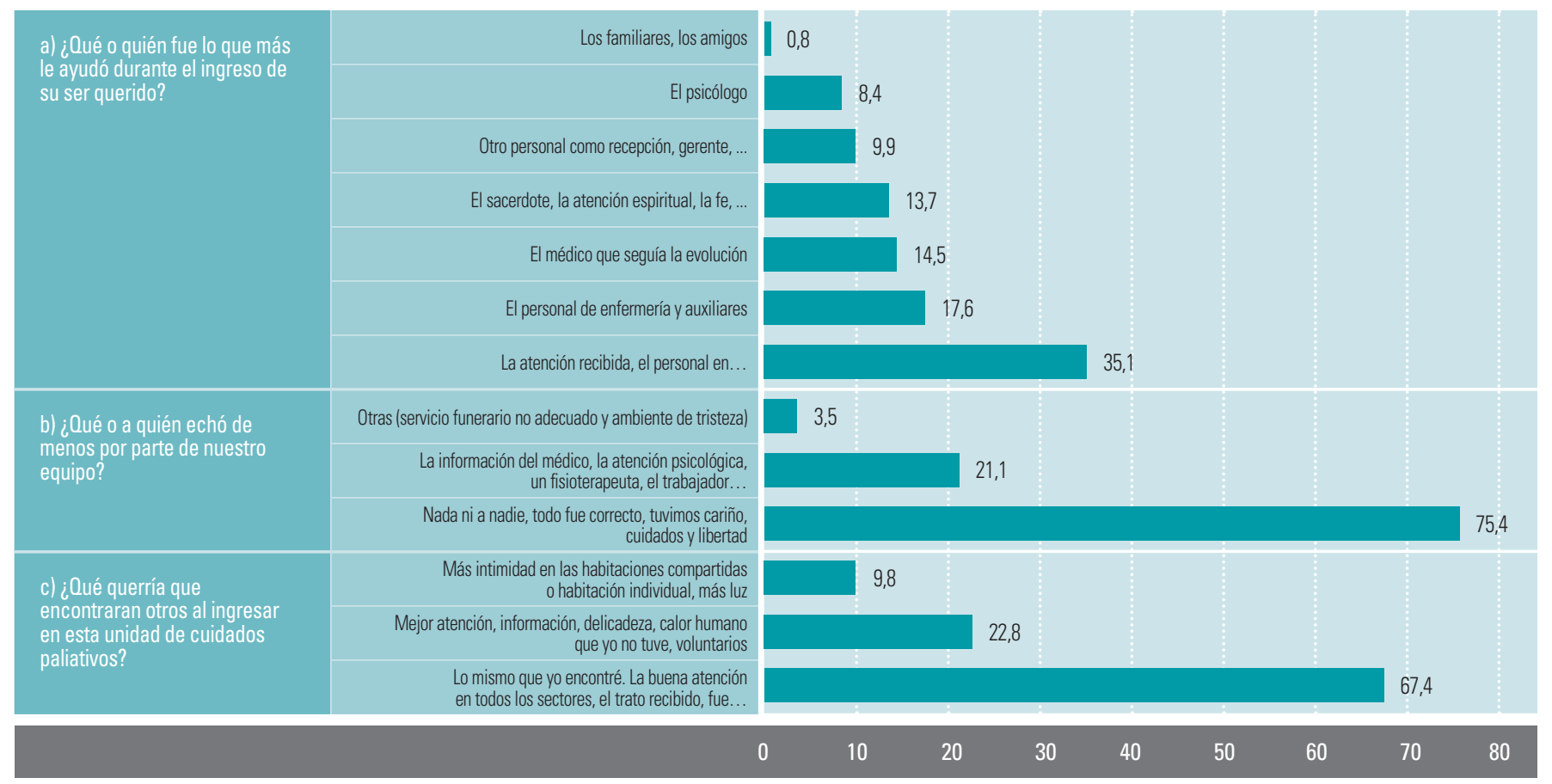

Figura 1. Respuestas a las preguntas abiertas (porcentajes de respuesta).

un patrón similar $50 \%$ en ambos estudios dicen no tener temas pendientes, $29 \%$ frente a $14 \%$ dicen haber recibido ayuda en este sentido, $15 \%$ frente a $37 \%$ no y $5 \%$ frente a $13 \%$ no saben o no contestan) $)^{3}$.

Respecto a las valoraciones del ambiente del equipo, si bien en el estudio de referencia ${ }^{3}$ la mayor puntuación de registros se localizó en la amabilidad (84\%), en el presente estudio han sido las características de acogedor (80\%) y amabilidad (77\%), a continuación tranquilizador y simpático $(68$ y $33 \%)$ y por último, al igual que en aquel3 ${ }^{3}$, sumando alrededor del $10 \%$ de las respuestas triste, áspero, frío y angustiante.

Esta similitud en los patrones confiere validez a ambos estudios como al estudio de Santacruz y cols. ${ }^{4}$ que también sigue estos niveles de satisfacción.

Respecto a los motivos de satisfacción, podríamos decir que estos hacen referencia al buen trato recibido, exquisito, aseo, comida, amabilidad y empatía, delicadeza, calor humano y voluntarios, y en general las instalaciones. Y los motivos de insatisfacción están orientados a la falta de información y la falta de atención de distintos profesionales (psicólogo, fisioterapeuta, trabajador social y sacerdote).

Más allá de los aspectos cuantitativos, nos parece interesante remarcar la importancia ${ }^{6}$ de lo que los familiares han concretado bajo los campos abiertos, que otros estudios podrían recoger bajo el epígrafe comentarios y sugerencias ${ }^{3,4}$ y que contribuyen a definir los factores que integran la calidad de vida en una UCP. Así, constatamos la importancia para el usuario de la facilitación o cobertura de aspectos que en principio pertenecen a la esfera íntima de las personas o son intangibles, como el trato recibido del personal, incluido cualquier tipo de personal, los ya debatidos (médico, auxiliar, enfermero) como los no tan nombrados, recepción, gerente, limpieza, voluntarios y sacerdotes. Incluso la simple sensación de presencia es muy importante. Y, parece que cuando se puntúa con valoraciones altas, lo que sostienen esos números es el cariño, cuidados y libertad, amabilidad y empatía, delicadeza y calor humano.

También aspectos más tangibles, la intimidad en las habitaciones y más luz, aseo, comida, y de cordialidad o educación, buena atención en todos los sectores. Es importante destacar que existe un porcentaje de personas que siguen echando de menos aspectos tan debatidos ya como la información del médico, la atención psicológica, u otros como un fisioterapeuta, trabajador social y sacerdote.

Este trabajo nos sirve para conocer más de cerca cómo ha sido la vivencia de los familiares de los enfermos que han fallecido recientemente en la UCP, para destacar la importancia de la asistencia integral y de cómo los aspectos más humanos y propios de la esfera íntima, lejos de resultar invasivos para las personas en situación de vulnerabilidad, son los que más se agradecen o más se echan de menos cuando no se reciben

\section{- BIBLIOGRAFÍA}

1. Sociedad Española de Cuidados Paliativos (SECPAL). www.secpal.es

2. Centro de Humanización de la salud (CEHS). www. humanizar es

3. Palomar C, Jiménez A, Barallat E, Juvero MT, Pérez E, Canal J, et al. Satisfacción de los familiares atendidos por un equipo de soporte en un hospital universitario. Med Pal. 2009;16:328-33.

4. Santacruz V, Carbonell JM. Evaluación de la satisfacción de los usuarios en cuidados paliativos. Med Pal. 1999;6:10-9.

5. Molina JM, Romero J, Romero Y, Rodríguez RM, Gálvez R. Encuesta de satisfacción a cuidadores en una unidad de cuidados paliativos. Med Pal. 2006;13:11-7.
6. Hauser JM, Kramer BJ. Family caregivers in palliative care. Clin Geriatr Med. 2004;20:671-88.

7. Harding $\mathrm{R}$, Higginson IJ. What is the best way to help caregivers in cancer and palliative care? A systematic literature review of interventions and their effectiveness. Palliat Med. 2003;17:63-74.

8. Higginson I, Wade A, McCarthy M. Palliative Care: view's of patients and their families. Br Med J. 1990:301:277-81.

9. Rodríguez A, Roca Casademont R, Planes M, Gras ME, Bayes R. Percepción subjetiva del paso del tiempo en el enfermo y el cuidador primario en cuidados paliativos hospitalarios. Med Pal. 2008;15:200-4.
10. Jurado MA, Esteve R. Dolor y sufrimiento en enfermos oncológicos al final de la vida: el papel del conocimiento de la enfermedad. Med Pal. 2009:16:229-34.

11. Jurado MA, Esteve R, Villalba 0. Causas de sufrimiento en enfermos oncológicos al final de la vida. Med Pal. 2010;17:90-5

12. Bayés $R$, Limonero JT, Barreto P, Comas MD. A way to screen for suffering in palliative care. J Palliat Care. 1997:3:22-6.

13. Uceda ME, Rodríguez JN, Mora Jl. Estudio piloto sobre aspectos familiares y sociales percibidos por cuidadores principales de pacientes oncológicos en cuidados paliativos domiciliarios. Med Pal 2007;14:69-74. 